\title{
Mechanisms of Vascular Endothelial Growth Factor-Induced Pathfinding by Endothelial Sprouts in Biomaterials
}

\author{
Amir Shamloo, Ph.D., ${ }^{1}$ Hui Xu, Ph.D., ${ }^{2}$ and Sarah Heilshorn, Ph.D. ${ }^{2}$
}

A critical property of biomaterials for use in regenerative medicine applications is the ability to promote angiogenesis, the formation of new vascular networks, to support regenerating tissues. Recent studies have demonstrated that a complex interplay exists between biomechanical and biochemical regulators of endothelial cell sprouting, an early step in angiogenesis. Here, we use a microfluidic platform to study the pathfinding behaviors induced by various stable vascular endothelial growth factor (VEGF) gradients during sprouting morphogenesis within biomaterials. Quantitative, time-lapse analysis of endothelial sprouting demonstrated that the ability of VEGF to regulate sprout orientation during several stages of sprouting morphogenesis (initiation, elongation, and turning navigation) was biomaterial dependent. Identical VEGF gradients induced different types of coordinated cell movements depending on the density of the surrounding collagen/fibronectin matrix. In denser matrices, sprouts were more likely to have an initial orientation aligned parallel to the VEGF gradient. In contrast, in less dense matrices, sprouts were more likely to initially misalign with the VEGF gradient; however, these sprouts underwent significant turning and navigation to eventually reorient to be parallel to the VEGF gradient. These less dense matrices required shallower VEGF gradients and demonstrated lower activating VEGF thresholds to induce proper sprout alignment and pathfinding. These results encourage the future use of microfluidic platforms to probe fundamental aspects of matrix effects on angiogenesis, to screen biomaterials for angiogenic potential, and to design ex vivo tissues with aligned vascular networks.

\section{Introduction}

A NGIOGENESIS, THE SPROUTING of new blood vessels from existing conduits, is a key requirement for the successful integration of implanted cells and biomaterials with host tissue. ${ }^{1}$ As scientists continue to develop new functional biomaterials with tunable biomechanical and biochemical properties for a myriad of applications, ${ }^{2-6}$ the effects of these materials on new blood vessel formation should also be considered. ${ }^{7-13}$ Both biomechanical and biochemical properties have been demonstrated to impact the ability of biomaterials to support blood vessel sprout formation and stabilization. $^{14-18}$

We have previously reported development of a microfluidic platform that produces stable soluble growth factor gradients for studies of endothelial cell (EC) sprouting within three-dimensional (3D) biomaterial matrices (Fig. 1A). ${ }^{19}$ In this previous work, we demonstrated that matrix density modifies sprout morphology (i.e., sprout width and lumen formation) within a single vascular endothelial growth factor (VEGF) concentration profile. ${ }^{19}$ Here, we couple our microfluidic platform with multi-day, time-lapse microscopy to study multiple VEGF concentration profiles for their ability to induce the dynamic pathfinding of EC sprouts within 3D biomaterials. We demonstrate that stable VEGF gradients can induce significant sprout turning behavior in elongating EC sprouts, which is highly reminiscent of the navigation strategy employed by neuronal growth cones in gradients of neurotrophic factors. ${ }^{20,21}$ Further, our results demonstrate that as the matrix density of a collagen/fibronectin biomaterial is increased, the minimum threshold of VEGF required to direct sprout orientation and navigation also increases.

During angiogenesis, ECs migrate away from existing blood vessels, organize into multi-cellular, capillary-like structures called sprouts, and are stabilized by additional cell types to form new blood vessels. ${ }^{22}$ In vivo, ECs lining the inside of conduit vessels experience the two-dimensional (2D) environment of the luminal surface; however, when they migrate into the surrounding tissue during angiogenesis, they experience the 3D environment of the surrounding extracellular matrix (ECM), which may include an implanted biomaterial. The degradation of the local ECM by proteases is the first step during this process. ${ }^{23-25}$ Outgrowth of endothelial tip cells into the matrix is mediated by 3D cellmatrix interactions and is followed by the proliferation of endothelial stalk cells. ${ }^{26,27}$ This coordinated migration and

Departments of ${ }^{1}$ Mechanical Engineering and ${ }^{2}$ Materials Science and Engineering, Stanford University, Stanford, California. 
proliferation of tip and stalk cells, respectively, forms hollow multi-cellular structures in the form of conduits with open lumens. ${ }^{22,26}$ The nascent sprouts further mature and are stabilized by the formation of tight adhesion junctions between the ECs as well as the recruitment of pericytes on the newly formed conduits. ${ }^{22,28}$

Multiple biochemical and biomechanical factors have been reported as directing this process. The spatiotemporal concentration distribution of soluble biochemical factors has been widely implicated as a key requirement in inducing angiogenesis. ${ }^{29}$ VEGF is a critical angiogenic regulator that can induce the formation and directional sprouting of ECs in vitro and in vivo. ${ }^{30}$ Recent studies have also shown that local distributions of soluble VEGF receptor-1 (VEGFR1) can control the directionality of EC sprouts, possibly due to its effect on local VEGF distribution around ECs. ${ }^{31}$ Besides soluble growth factors, the ECM also plays a critical role in organizing ECs during capillary morphogenesis. ${ }^{28}$ The ECM provides structural support and adhesion sites for ECs that are required for cell survival, proliferation, and migration. ${ }^{28}$ The ECM also serves as an anchor point for multiple tethered biochemical factors that can initiate intracellular signaling cascades. ${ }^{32}$ Additionally, the mechanical properties of the ECM can control the tension forces exerted across the cell body. ${ }^{33,34}$ Recent studies have demonstrated that matrix mechanical stiffness can mediate sprout formation ${ }^{18,35}$ and morphology, ${ }^{19,34}$ with more elongated sprouts observed within compliant matrices. ${ }^{18,19,34}$ Although much is still unknown about how these mechanical forces are translated into biochemical signals, recent studies have demonstrated that ECs modify their transcription factor regulation in response to ECM mechanics, thus resulting in altered levels of VEGFR2 in vitro and in vivo. ${ }^{14}$ These results suggest there is a complex interplay between the biomechanical and biochemical extracellular signals that regulate angiogenesis, and both sets of factors should be simultaneously considered when developing implantable biomaterials for regenerative medicine therapies and when developing in vitro models of angiogenesis.

Although many in vitro EC-biomaterial studies are performed in 2D to mimic the endothelial lining of the blood vessel lumen, ${ }^{36-38}$ angiogenic sprouting occurs within a 3D microenvironment. Cell morphology and function as well as signal transduction and gene expression are known to be quite different in 2D versus 3D environments. ${ }^{39}$ Many of the previous platforms used for 3D studies of angiogenesis have implemented fibrin gel as the surrounding matrix. ${ }^{12,16,40-42}$ Applying optimized VEGF gradients within alginate biomaterial scaffolds was shown to enhance the treatment of ischemic tissues by directing EC sprout formation. ${ }^{16}$ Recently, there have been studies of angiogenesis within microfluidic devices using collagen matrix as a 3D biomaterial to probe the role of interstitial fluid flow and supporting cell types in regulating angiogenesis. ${ }^{43-45}$ In all these previous 2D and 3D studies, evidence of EC sprout turning was not reported, and sprout navigation within stable growth factor gradients was not quantitatively investigated. Here, we utilize collagen matrices of varying density (all supplemented with equal amounts of fibronectin) to investigate the interplay between matrix density and VEGF concentration profile in regulating the alignment and pathfinding of EC sprouts. In-depth understanding of the mechanisms used by EC sprouts to undergo sprout navigation may be useful in future applications of in vitro gradients to design prevascularized biomaterials to promote EC sprout alignment and anastomosis. $^{46}$

\section{Materials and Methods}

\section{Microfluidic device fabrication}

Soft lithography techniques were used to fabricate the devices as previously described. ${ }^{19,38}$ Briefly, two layers of fluidic channels were designed by AutoCad software and patterned on transparency masks. The Stanford Microfluidics Foundry fabricated a silicon wafer master mold by using negative photoresist (SU-8). Polydimethylsiloxane (PDMS; Sylgard 184) was used to fabricate the devices by using the silicon mold treated with chlorotrimethylsilane (Sigma). Inlets and outlets were punched by using sharpened needles (20 gauge). To adhere the device to glass, both surfaces were exposed to oxygen plasma for 2-3 min and irreversibly bound together.

\section{Human dermal microvascular EC culture}

Adult human dermal microvascular endothelial cells (HDMVEC; Lonza) were grown in endothelial growth medium-2 MV (EGM-2 MV, 5\% serum; Lonza). Media was changed every 2 days, and cells were kept in a humidified, $5 \% \mathrm{CO}_{2}$ environment at $37^{\circ} \mathrm{C}$. HDMVEC were nonenzymatically passaged by using EDTA solution (Gibco). Passages 3-8 were used. Microcarrier dextran beads ( $\mathrm{d} \sim 170 \mu \mathrm{m}$, Cytodex-3; GE Healthcare) were hydrated in phosphate-buffered saline (PBS) and autoclaved. The sterilized solution was allowed to separate in a well plate, and the supernatant was decanted. Beads were rinsed twice in EGM$2 \mathrm{MV}$ and gradually added to a $10-\mathrm{cm}$, nontissue culture plate to homogeneously cover the plate surface. HDMVEC $\left(7 \times 10^{6}\right.$ cells $/ 10-\mathrm{cm}$ plate) were incubated with the beads on a platform shaker inside the incubator for $3-4 \mathrm{~h}$. After cell adhesion to the beads, they were transferred to a fresh sixwell plate and allowed to settle before the supernatant was replaced with $2 \mathrm{~mL}$ of fresh serum-free medium. To estimate the bead concentration, a specified volume of medium was placed on a slide, and beads were counted by using phase contrast microscopy.

\section{Endothelial sprout pathfinding studies}

Stock collagen (rat tail collagen I; BD Biosciences) was mixed with fibronectin (final concentration of $5 \mu \mathrm{g} / \mathrm{mL}, 10 \%$ total volumetric mixture) and microcarrier beads (final concentration of $\sim 20$ beads/device) and transformed into the gel phase by the addition of sodium hydroxide $(0.5 \mathrm{~N}$ stock solution, $5 \%$ volumetric mixture with stock collagen). Different densities of collagen matrix were made while keeping the final fibronectin, microcarrier bead, and media concentrations uniform. Immediately after mixing, $\sim 20 \mu \mathrm{L}$ of the solution was injected into the cell culture chamber of the device; gelation occurred in 5-10 min. Both ends of the cell culture chamber were pin-plugged, and the device was covered with media to avoid evaporation. EGM-2 MV supplemented with various concentrations of VEGF (VEGF-A isoform VEGF(165); R\&D Systems) was continuously supplied to the source reagent channel, whereas EGM-2 MV 
with no VEGF was supplied to the sink reagent channel. The injection flow rate was $40 \mathrm{~nL} \mathrm{~min}^{-1}$. The entire system (microfluidic device, syringe pump, and syringes) was placed in the incubator during the 4 days of culture. Syringes were filled with fresh media daily.

\section{Concentration gradient quantification}

Tubing (Upchurch Scientific) was inserted into the inlets of the reagent channels and connected to $100 \mu \mathrm{L}$ syringes (Hamilton) mounted on a syringe pump (World Prerecision Instruments). To measure the reagent concentration distribution inside the cell culture chamber, two tracers were used: (i) Dextran/Texas Red (MW $=20 \mathrm{kDa}$; Sigma) with a similar molecular weight to the protein VEGF-165 (MW =20 kDa) and (ii) VEGF-165 conjugated with FITC (VEGF-165/FITC). VEGF conjugation was performed with a FITC labeling kit (EMD Chemicals) following manufacturer's instructions. Briefly, VEGF and FITC were mixed at a 1:60 molar ratio, incubated at room temperature for $2 \mathrm{~h}$ in the dark, and dialyzed at $4^{\circ} \mathrm{C}$ against PBS for $24 \mathrm{~h}$. Cell culture media were dosed with VEGF-165/FITC (800 ng/mL) and Dextran/Texas Red $(1 \mu \mathrm{M})$. A fluorescent inverted microscope (Zeiss) with a CCD camera was used for imaging. MATLAB software was used to measure the fluorescence intensity of each image pixel inside the cell culture chamber and to normalize against the fluorescence of solutions of known concentrations.

\section{Quantification of sprout pathfinding and statistical analysis}

Individual beads were imaged every $24 \mathrm{~h}$ for 4 days by using phase contrast and fluorescence microscopy. For some trials, cells were fluorescently labeled with Cell Tracker CMFDA $(2 \mu \mathrm{g} / \mathrm{mL}, 20-\mathrm{min}$ incubation; Molecular Probes) before mixing with microcarrier beads. Micrographs were analyzed by hand using ImageJ software (NIH freeware) to measure the number of sprouts per bead, the sprout initiation angle $(\phi$, see Fig. $3 C)$, the starting elongation angle $\left(\theta_{1}\right.$, see Fig. $3 C$ ), and the final elongation angle $\left(\theta_{2}\right.$, see Fig. $\left.3 C\right)$. The Sprout Turning Index (STI) was defined as: $\mathrm{STI}=\left(\theta_{1}-\right.$ $\left.\theta_{2}\right) / \theta_{1}$. During time-lapse imaging over multiple days, the starting elongation angle $\left(\theta_{1}\right)$ did not appreciably change; therefore, both the starting and final elongation angles could be measured from a single image at the end of each experiment. For each condition reported, at least $n=3$ independent experiments were performed, and $\sim 20$ beads per device were imaged in each experiment. All data are reported as averages \pm standard deviations of the independent experiments for each condition. One-tailed, nonpaired, Student's $t$-test was used to determine the statistical significance of differences between pairs of conditions.

\section{Results}

\section{Multi-day, time-lapse imaging of sprout elongation}

To study the effects of matrix density on VEGF-induced endothelial sprout pathfinding, adult HDMVECs were cultured within 3D matrices inside a microfluidic device that generates concentration gradients of soluble biochemical factors which remain indefinitely stable (Fig. 1A). ${ }^{19}$ All matrices included a constant supplement of $5 \mu \mathrm{g} / \mathrm{mL}$ fibronectin and varying collagen densities of $0.7,1.2,1.9$, and $2.7 \mathrm{mg} / \mathrm{mL}$.
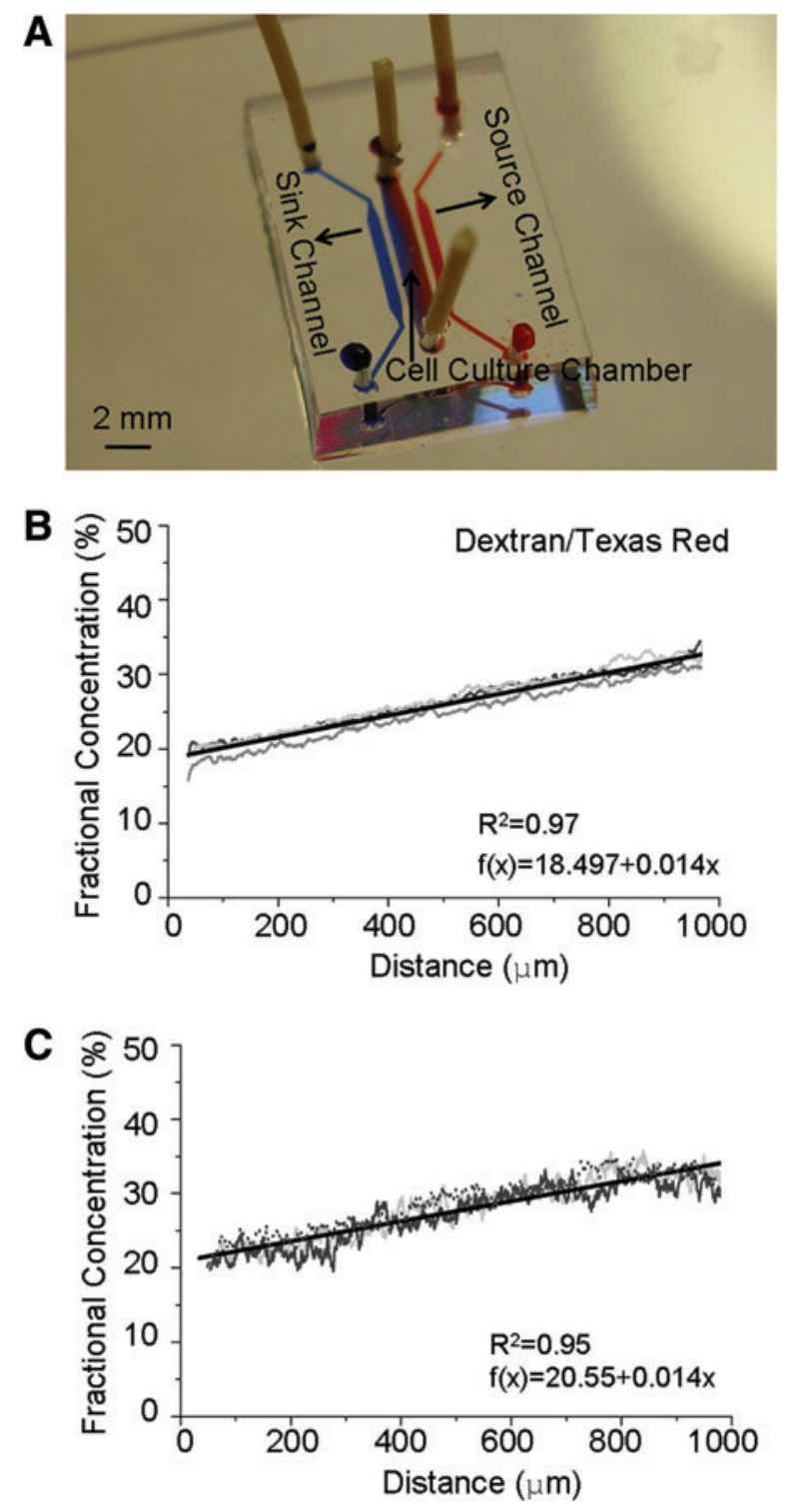

FIG. 1. Microfluidic gradient generator device. (A) Photograph of device filled with dye for visualization of gradient. Microcapillaries (not visible in the image) connect the source and sink channels to the cell culture chamber, enabling formation of a stable gradient across the cell culture chamber. B-C. Quantification of equilibrium gradients of Dextran/ Texas Red (B) and VEGF-165/Texas Red (C) in the highdensity biomaterial $(5 \mu \mathrm{g} / \mathrm{mL}$ fibronectin and $2.7 \mathrm{mg} / \mathrm{mL}$ collagen). For each condition, three individual fluorescence intensity measurements (thin gray lines) and a least-squares linear regression (thick black lines) are shown. VEGF, vascular endothelial growth factor. Color images available online at www.liebertonline.com/tea

As previously reported, higher density matrices were stiffer and had higher plateau storage moduli $\left(G^{\prime}\right) .{ }^{19}$ Within the microfluidic device, all matrix densities resulted in identical stable, linear VEGF concentration profiles within at least 2 h. ${ }^{19}$ Importantly, the quantified gradients as observed using FITCconjugated VEGF and Texas Red-conjugated dextran were identical (Fig. 1B, C), thus demonstrating negligible binding between the soluble VEGF and the fibronectin/collagen biomaterials. 
Table 1. Parameters of Equilibrium Vascular Endothelial Growth Factor Profiles

\begin{tabular}{lccc}
\hline & $\begin{array}{c}\text { [VEGF }]_{\text {min }} \text { in } \\
\text { culture chamber } \\
(n g / m L)\end{array}$ & $\begin{array}{c}{[\text { VEGF }]_{\text {max }} \text { in }} \\
\text { culture chamber } \\
(n g / m L)\end{array}$ & $\begin{array}{c}\text { [VEGF] } \\
\text { gradient } \\
(n g / m L / m m)\end{array}$ \\
\hline 1 & 97 & 143 & 46 \\
2 & 123 & 146 & 23 \\
3 & 48 & 71 & 23 \\
\hline
\end{tabular}

To probe the dynamics of sprout elongation and pathfinding, we performed time-lapse imaging microscopy over four days in biomaterials subjected to three different VEGF concentration profiles (Table 1). In Profile 1, the maximum VEGF concentration within the matrix was set to be $\sim 150 \mathrm{ng} / \mathrm{mL}$, which is slightly higher than the reported minimum concentration threshold to induce EC sprouting, ${ }^{16,19}$ with a gradient steepness of $\sim 50 \mathrm{ng} / \mathrm{mL} / \mathrm{mm}$. In addition to the VEGF profile just described (Profile 1), two profiles were formed with gradients approximately half as steep. In Profile 2, the maximum concentration was designed to be similar to Profile 1, whereas in Profile 3, the maximum concentration was approximately half of that in Profile 1.

Similar to our previous studies, ECs were first cultured as 2D monolayers on the surfaces of dextran microcarrier beads $(\mathrm{d} \sim 170 \mu \mathrm{m})$; the cell-coated beads were then encapsulated within the matrix and injected into the microfluidic device. On stimulation with a stable soluble gradient of VEGF, the ECs undergo collective cell migration away from the bead surfaces and into the biomaterial matrix to induce endothelial sprout formation. This encapsulated bead geometry mimics the $2 \mathrm{D}$ to $3 \mathrm{D}$ microenvironment transition experienced by ECs during in vivo angiogenesis and has been widely utilized as an in vitro model of sprouting morphogenesis. ${ }^{29,41,42,47}$ Within the lowest-density collagen matrix $\left(0.7 \mathrm{mg} / \mathrm{mL}, \mathrm{G}^{\prime} \sim 30 \mathrm{~Pa}\right)$, tracks of multiple cells began to coordinate their migration to form unstable sprout structures within $24 \mathrm{~h}$ (Fig. 2A). Within biomaterials with intermediate collagen density $\left(1.2 \mathrm{mg} / \mathrm{mL}, \mathrm{G}^{\prime} \sim 80 \mathrm{~Pa}\right)$, ECs formed a few small clusters after $24 \mathrm{~h}$ by migrating on the bead surfaces in
A
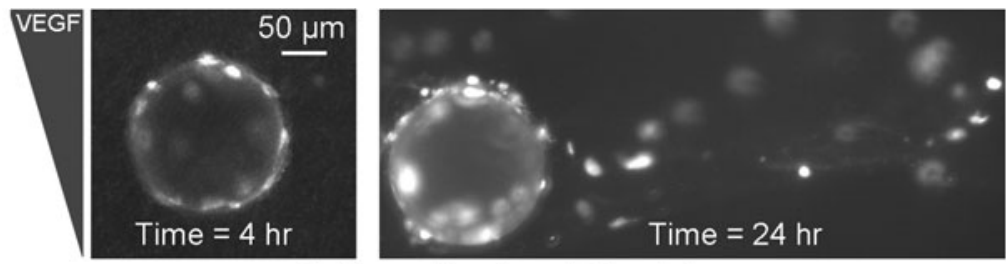

Collagen density $=0.7 \mathrm{mg} / \mathrm{ml}$

B
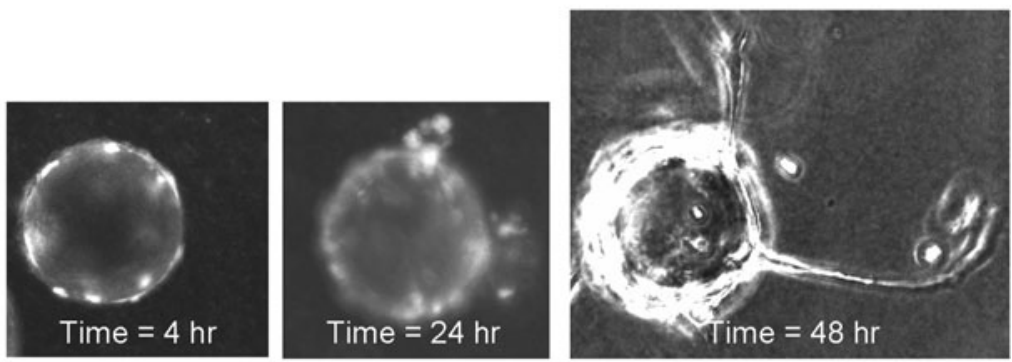

Collagen density $=1.2 \mathrm{mg} / \mathrm{ml}$

C
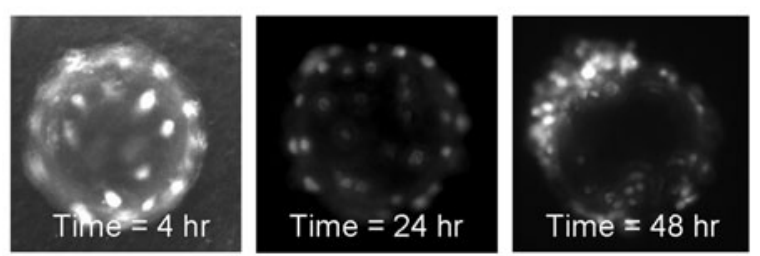

Collagen density $=1.9 \mathrm{mg} / \mathrm{ml}$

D
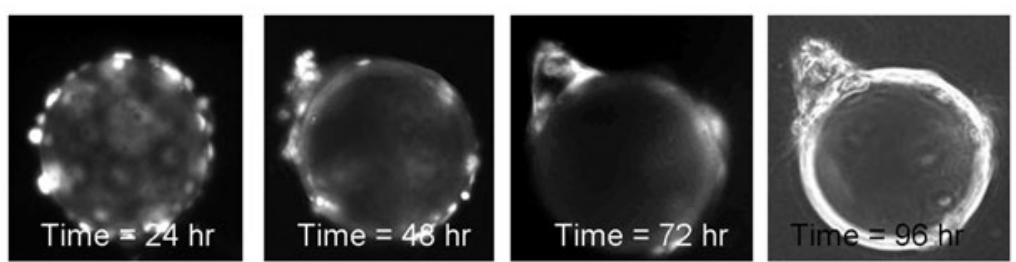

Collagen density $=2.7 \mathrm{mg} / \mathrm{ml}$
FIG. 2. Representative time-lapse images of endothelial sprouting morphogenesis in matrices with collagen densities of $0.7,1.2$, 1.9 , and $2.7 \mathrm{mg} / \mathrm{mL}$ (A-D, respectively) and identical equilibrium VEGF gradients (Profile 1). Phase contrast micrographs are shown for the final time points in B-D. All other images are fluorescent micrographs of Cell Tracker labeled cells. 
a 2D manner before exploration of the 3D matrix environment. Several of these clusters were able to form elongated stable sprouts after one to two more days in culture (Fig. 2B). Within biomaterials with a higher collagen gel density $\left(1.9 \mathrm{mg} / \mathrm{mL}, \mathrm{G}^{\prime}\right.$ $\sim 200 \mathrm{~Pa}$ ), ECs were observed as undergoing less migration and clustering on the bead surfaces after $24 \mathrm{~h}$ compared with lower density matrices; however, by $48 \mathrm{~h}$, defined EC clusters had formed on the bead surfaces. These clusters finally formed stable, thick sprouts within the matrix after about $72 \mathrm{~h}$ (Fig. 2C). The cells within the highest density matrices $(2.7 \mathrm{mg} / \mathrm{mL}$, $\mathrm{G}^{\prime} \sim 700 \mathrm{~Pa}$ ) also showed a delayed polarization toward the VEGF gradient on the bead surfaces, and they were generally unable to elongate into the matrix and instead formed clusters of cells on the bead surfaces (Fig. 2D). Across multiple independent trials and consistent with our previous observations, ${ }^{19}$ the time required for new sprouts to assemble was found to increase within biomaterials of increasing density.

\section{Time-lapse imaging and quantification of sprout pathfinding}

Previous studies of 3D sprouting have found that ECs specialize into highly migratory tip cells at the leading edge of sprouts and proliferative stalk cells along the sprout length. ${ }^{26}$ The tip cell, which may up-regulate VEGFRs on specialization, ${ }^{48}$ is hypothesized to be the critical gradientsensing element of the sprout and to dictate overall sprout elongation and pathfinding. ${ }^{26}$ By following a single sprout for several days, we observed significant sprout pathfinding within the VEGF gradient. For example, after $48 \mathrm{~h}$ in a $1.2 \mathrm{mg} / \mathrm{mL}$ density matrix exposed to VEGF Profile 1 , a previously misaligned sprout performed a tight turn $\left(\sim 90^{\circ}\right)$ to align parallel to the gradient (Fig. 3A, B).

Three different mechanisms of pathfinding were observed during sprouting morphogenesis. First, the ECs were observed as migrating on the surface of the bead and forming a sprout at a specific initiation point. Pathfinding due to polarization of sprout initiation was analyzed by measuring the angle between the VEGF gradient and the sprout initiation point ( $\phi$, Fig. 3C). Second, the newly initiated sprouts were observed as leaving the bead surface and following an initial linear trajectory. To quantify this navigation, we defined the starting elongation angle $\left(\theta_{1}\right)$ as the angle between the VEGF gradient and the sprout longitudinal axis along the first $50 \mu \mathrm{m}$ of the sprout length (Fig. 3C). Third, sprouts were observed as turning and changing the direction of their elongation trajectory. This pathfinding mechanism was quantified by measuring the final elongation angle $\left(\theta_{2}\right)$, that is, the angle between the VEGF gradient and the longitudinal axis along the last $50 \mu \mathrm{m}$ of the sprout length. This quantification was performed for all three VEGF profiles in the two collagen densities that resulted in the greatest number of sprouts $(1.2$ and $1.9 \mathrm{mg} / \mathrm{mL})$.

\section{Denser matrices require steeper VEGF gradients to polarize sprout initiation}

To evaluate the sprout initiation point, the beads were divided into two hemispheres facing higher $\left(\phi=0^{\circ}-180^{\circ}\right)$ or lower $\left(\phi=180^{\circ}-360^{\circ}\right)$ VEGF concentrations. By comparing the number of sprouts initiated on each side of the bead, we obtained a quantitative measure for the bead polarity. We previously reported that VEGF Profile 1 was capable of polarizing sprout initiation in both 1.2 and $1.9 \mathrm{mg} / \mathrm{mL}$ density matrices. ${ }^{19}$ Here, we vary the VEGF concentration profile for comparison with our previous results and to evaluate the effects of absolute VEGF concentration and VEGF gradient steepness on sprout polarization. In the lower density matrices (density $=1.2 \mathrm{mg} / \mathrm{mL}$ ), the total percentage of beads with sprouts (the summation of the two columns, Fig. 4A) is significantly less in VEGF Profile 3 compared with Profiles 1 and 2, which have higher VEGF maximum concentrations. However, for all three VEGF profiles, significantly more sprouts were initiated from the half of the bead subjected to higher VEGF concentrations $(p<0.05)$. These results suggest that in lower density collagen matrices, gradients of $\sim 25 \mathrm{ng} / \mathrm{mL} /$ $\mathrm{mm}$ are sufficient to polarize the location of sprout initiation.

Within higher density matrices (density $=1.9 \mathrm{mg} / \mathrm{mL}$ ), a reduction in the total number of sprouts was also observed in Profile 3 compared with Profiles 1 and 2 (Fig. 4B). In addition, Profile 3 was not effective in polarizing the sprout initiation point. Increasing the VEGF maximum concentration, but still keeping the VEGF gradient identical ( 25 ng/mL/ $\mathrm{mm}$ ), increased the total number of sprouts but did not induce a significant polarization of sprout initiation. In contrast, increasing the VEGF concentration gradient to $50 \mathrm{ng} /$ $\mathrm{mL} / \mathrm{mm}$ induced a significant polarization of sprout initiation $(p<0.01)$. These results demonstrate that matrices with higher densities require steeper VEGF concentration gradients to induce polarization of the sprout initiation points.

\section{Tip cell navigation during elongation: sprout turning in VEGF gradients}

For sprouts within less dense collagen matrices (density $=1.2 \mathrm{mg} / \mathrm{mL}$ ), moderate orientation of the starting
FIG. 3. Time-lapse imaging of sprout pathfinding. (A) Representative phase contrast image of a misaligned sprout at $24 \mathrm{~h}$ in a matrix with collagen density $=1.2 \mathrm{mg} / \mathrm{mL}$ and VEGF Profile 1. Inset shows the tip of the sprout at higher magnification. (B) Fluorescent micrograph of Cell Tracker labeled cells. After $48 \mathrm{~h}$, the sprout in (A) has turned to align parallel to the VEGF gradient. (C) Schematic of sprout pathfinding image analysis: initiation $(\phi)$, starting elongation $\left(\theta_{1}\right)$, and final elongation $\left(\theta_{2}\right)$ angles relative to the VEGF gradient.
B
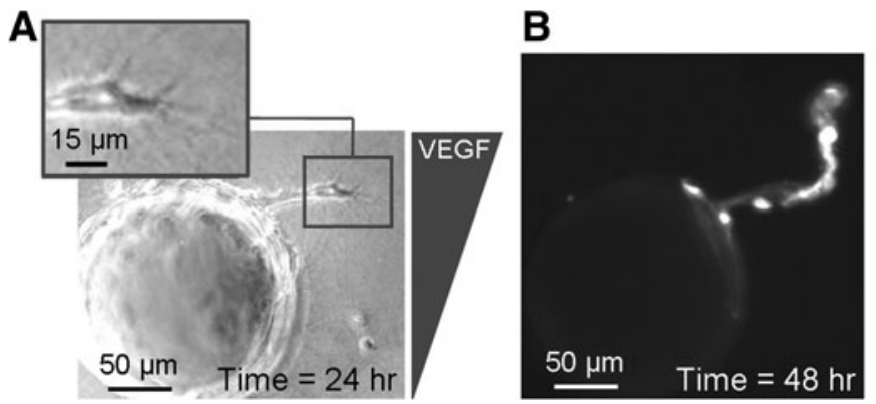

C

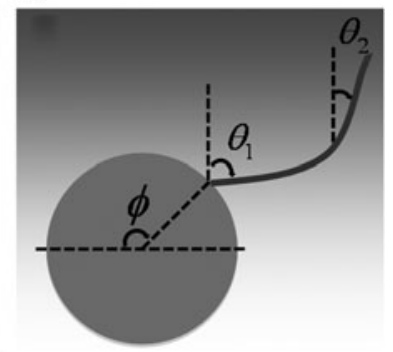


A

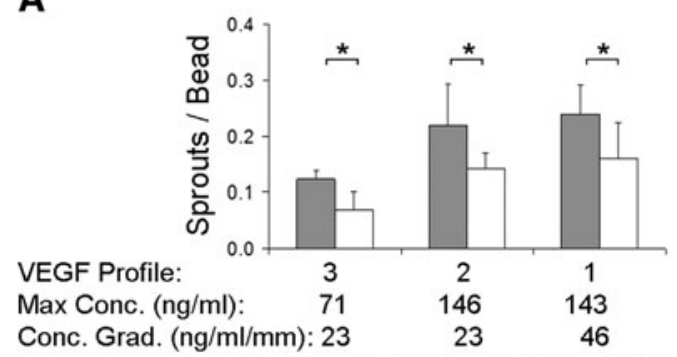

Collagen density $=1.2 \mathrm{mg} / \mathrm{ml}$
B

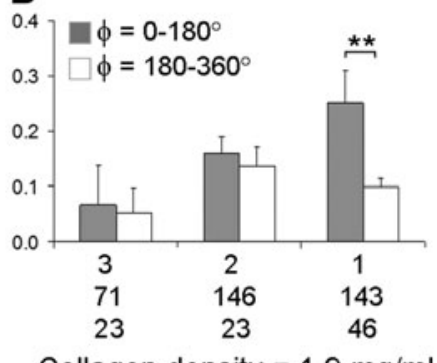

Collagen density $=1.9 \mathrm{mg} / \mathrm{ml}$
FIG. 4. Polarization of sprout initiation within stable VEGF gradients. (A, B) Distribution of sprout initiation points within matrices of collagen density $=1.2$ and $1.9 \mathrm{mg} / \mathrm{mL}$, respectively. The bead surface is bisected into two regions: facing toward higher $(\phi=0$ $180^{\circ}$, shaded bars) or lower $(\phi=180$ $360^{\circ}$, white bars) VEGF concentrations; $n \geq 3$ microdevices per condition, each containing $\sim 20$ beads, 4 days culture; ${ }^{*} p<0.05 ;{ }^{* *} p<0.01$. elongation angle parallel to the VEGF gradient was observed for all three VEGF concentration profiles (Fig. 5A). In contrast, in denser collagen matrices (density $=1.9 \mathrm{mg} / \mathrm{mL}$ ), the tendency of the starting sprout to align with the VEGF gradient was significantly dependent on the VEGF profile, with the steepest VEGF gradient (Profile 1) resulting in substantially more starting alignment (Fig. 5B). These findings demonstrate that denser collagen matrices can induce significant alignment in the early stages of sprout formation, but only if a sufficiently steep VEGF gradient is present.

Direct comparison of the starting and final elongation angles $\left(\theta_{1}\right.$ and $\left.\theta_{2}\right)$ is indicative of sprout tip navigation and turning during elongation. In the less dense collagen matrices, the distribution of final elongation angles demonstrates that the sprouts have undergone significant reorientation to strongly align with the VEGF gradient (Fig. 5A, C). Although this pathfinding behavior was observed in all VEGF profiles tested, the navigational response was most pronounced in the steepest VEGF concentration gradient, Profile 1. In marked contrast, in denser matrices, the distribution of final sprout elongation angles remained relatively unchanged compared with the starting elongation angle distributions for all three VEGF profiles (Fig. 5B, D). Therefore, sprouts that initially misaligned were less likely to reorient within denser matrices compared with less dense matrices.

To quantify this turning behavior, a new parameter was defined, the STI. The STI is defined as the ratio of the ob- served sprout turning angle to the maximum possible sprout turning angle to fully align with the VEGF gradient: STI= $\left(\theta_{1}-\theta_{2}\right) / \theta_{1}$. Therefore, an STI of 0 indicates no turning, and an STI of 1 indicates a turn that resulted in perfect alignment with the gradient. For all VEGF profiles tested, sprouts had a significantly higher turning capacity to reorient with the VEGF gradient within less dense matrices compared with denser matrices (Fig. 5E). In steeper VEGF gradients ( $\sim 50 \mathrm{ng} /$ $\mathrm{mL} / \mathrm{mm}$, Profile 1$)$, the average sprout was able to make a turn that was $\sim 45 \%$ of the maximal tuning angle. In contrast, in shallower VEGF gradients $(\sim 25 \mathrm{ng} / \mathrm{mL} / \mathrm{mm})$ regardless of the absolute VEGF concentration (Profiles 2 and 3 ), the STI was only $\sim 25 \%$. Therefore, in less dense matrices, the steepness of the VEGF gradient appears to be a more dominant factor in guiding tip cell navigation compared with the absolute VEGF concentration. Taken together, these findings demonstrate that two different mechanisms are employed to align sprouts within 3D biomaterials. In denser collagen matrices, steep VEGF gradients induce alignment during the early stages of sprout formation, with little observed deviation from the original direction during continued sprout elongation. In contrast, in less dense collagen matrices, steep VEGF gradients induce substantial sprout turning to reinforce proper navigation and sprout pathfinding. Therefore, identical VEGF gradients can induce various types of coordinated cell movements that are modulated by the density of the surrounding biomaterial matrix.
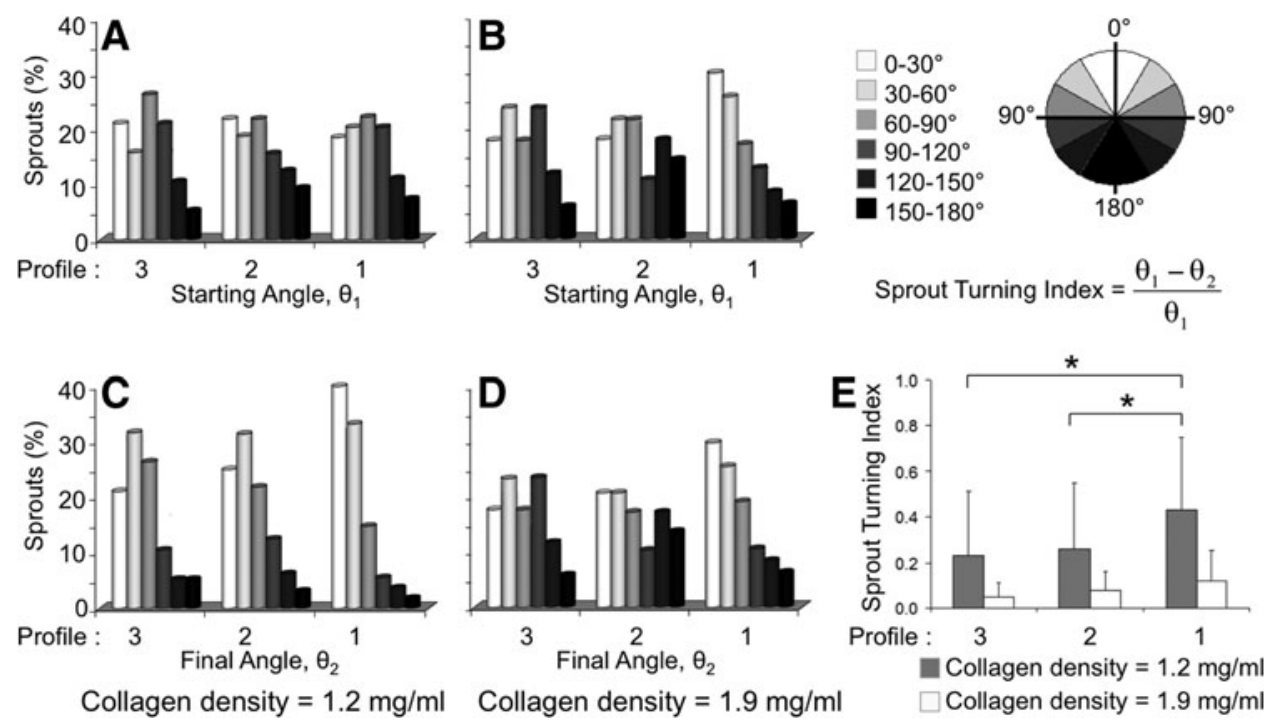

FIG. 5. Sprout pathfinding during elongation. Elongation angles are binned into six angular regions shown with increasing shadow intensity for lower VEGF concentrations. (A-D) Distribution of starting (A, B) and final (C, D) elongation angles in collagen matrices of lower (A, C, $1.2 \mathrm{mg}$ / $\mathrm{mL})$ and higher (B, D, $1.9 \mathrm{mg} /$ $\mathrm{mL}$ ) densities for three VEGF concentration profiles. Comparison of starting and final elongation angles is indicative of sprout turning. (E) Sprout Turning Index within selected microenvironments. For A-E, $n \geq 4$ microdevices per condition, each containing $\sim 20$ beads, 4 days culture; ${ }^{*} p<0.05$. 


\section{EC sprouts in denser matrices have higher VEGF activation and saturation thresholds}

Previous work has demonstrated that above a specific VEGF concentration, the effects on EC migration and sprouting become saturating as the majority of receptors become bound. ${ }^{16}$ In addition, a minimum VEGF concentration is required to elicit a cellular response. ${ }^{16,38}$ To quantify the potential threshold concentration effects of VEGF gradients on sprout pathfinding, the cell culture chamber of a device subjected to Profile 1 was divided into three zones transversely, with each zone having an identical gradient steepness of $\sim 50 \mathrm{ng} / \mathrm{mL} / \mathrm{mm}$. Zone I is furthest from the VEGF source, Zone II is in the middle, and Zone III is closest to the VEGF source, corresponding to average concentrations of 110,125 , and $140 \mathrm{ng} / \mathrm{mL}$, respectively (Fig. 6A). Within the lower density collagen matrices, the overall number of sprouts formed in each Zone was statistically identical (Fig. 7A). Similarly, the final sprout elongation angles $\left(\theta_{2}\right)$ were substantially aligned with the VEGF gradient in all three Zones (Figs. 6B-D and 7B). These results demonstrate that $110 \mathrm{ng} / \mathrm{mL}$ is above the activation threshold for VEGF in the lower density matrices and may be saturating. In contrast, within the higher density collagen matrices, the number of sprouts formed and the sprout orientation were significantly different across the three Zones (Fig. 6E-G). The incidence of sprouting was significantly increased at higher VEGF concentrations (Fig. 7C). Similar to the frequency data, final sprout alignment was strongly correlated to the VEGF concentration in the higher density matrices (Fig. 7D). Taken together, these data suggest that EC sprouts have higher VEGF activation and saturation thresholds in higher density biomaterial matrices compared with lower density biomaterials.

\section{Discussion}

In this study, 3D endothelial sprout navigation was examined by using a microfluidic platform that enabled the separate and simultaneous tuning of biomaterial parameters and soluble biochemical stimuli. Increasing the collagen density within a blended collagen/fibronectin matrix was observed as resulting in slower sprout initiation, altered sprout pathfinding behavior, and increased VEGF activation and saturation thresholds. The perturbation of a single scaffold variable (collagen density) impacts multiple biomaterial properties including diffusivity, matrix mechanics, fibril morphology, and presentation of cell-binding domains (each of which is briefly discussed next). This tendency to impact multiple material characteristics when making a single change in biomaterial formulation is quite common for both natural and synthetic polymeric biomaterials. ${ }^{4,5}$ This highly coupled complexity in material properties underscores the need for high-throughput, in vitro screening tools to help assess the angiogenic potential of various biomaterial formulations. Further, these results support the notion that no single VEGF concentration profile is optimal to support sprouting morphogenesis in all cases, but rather the ideal VEGF concentration profile will be dependent on the choice of biomaterial scaffold for each specific application.

Although increasing collagen density did result in retarded diffusivity of VEGF, all matrix formulations were observed as having similar equilibrium VEGF concentration profiles within $120 \mathrm{~min}$, a time-interval that is negligible
FIG. 6. Dependence of sprout pathfinding on average VEGF concentration. (A) Top-down schematic of the microfluidic device; the cell culture chamber is divided into 3 zones transversely. The VEGF average concentration and gradient within each zone is shown in the table. (B-D) Representative phase contrast images of sprouts initiated at Zones I-III, respectively, collagen density $=1.2 \mathrm{mg} / \mathrm{mL}, 4$ days culture. White arrows depict the orientation of the last $50 \mu \mathrm{m}$ of each sprout. (E-G) Representative images of sprouts initiated at Zones I-III, respectively, density $=1.9 \mathrm{mg} / \mathrm{mL}$.

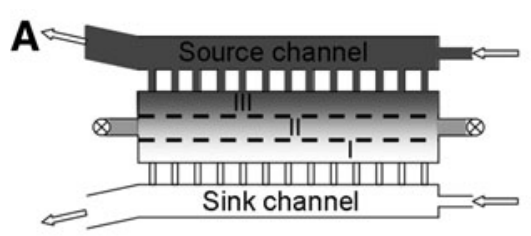

\begin{tabular}{|c|c|c|}
\hline Zone & $\begin{array}{c}\text { Ave. Conc. } \\
(\mathrm{ng} / \mathrm{ml})\end{array}$ & $\begin{array}{c}\text { Gradient } \\
(\mathrm{ng} / \mathrm{ml} / \mathrm{mm})\end{array}$ \\
\hline III & 140 & 46 \\
\hline II & 125 & 46 \\
\hline I & 110 & 46 \\
\hline
\end{tabular}

B

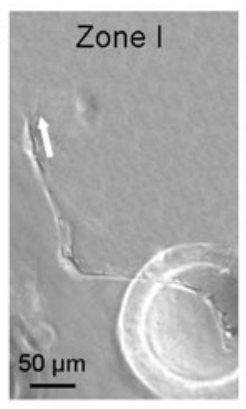

C

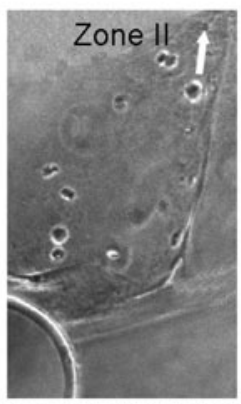

Collagen density $=1.2 \mathrm{mq} / \mathrm{ml}$

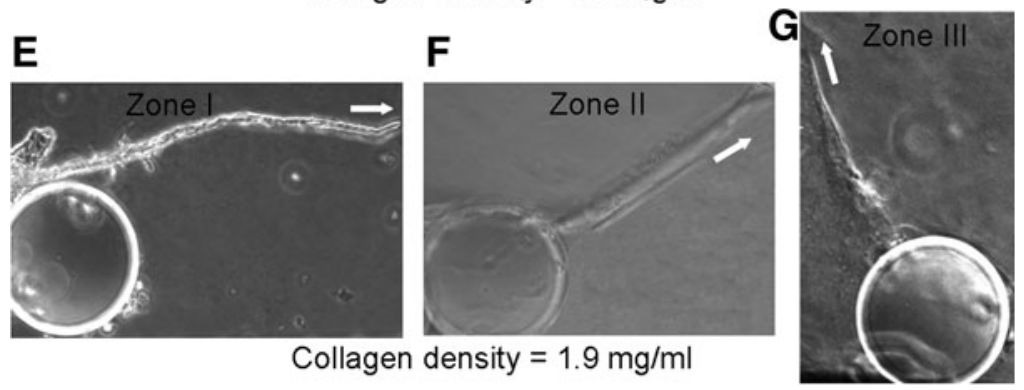


A
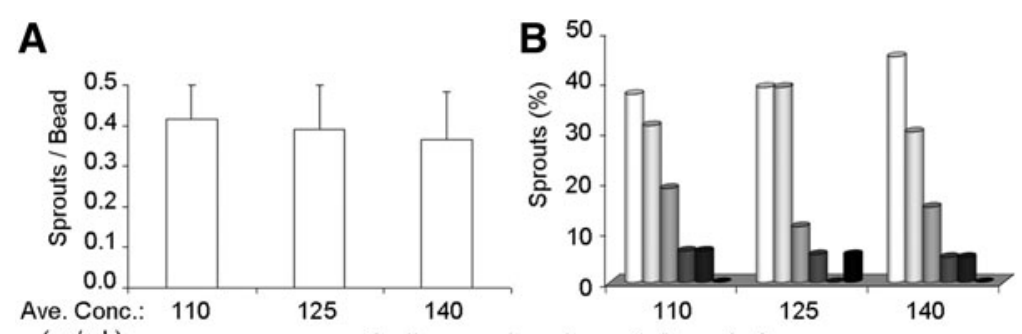

$(\mathrm{ng} / \mathrm{mL})$ Collagen density $=1.2 \mathrm{mg} / \mathrm{ml}$
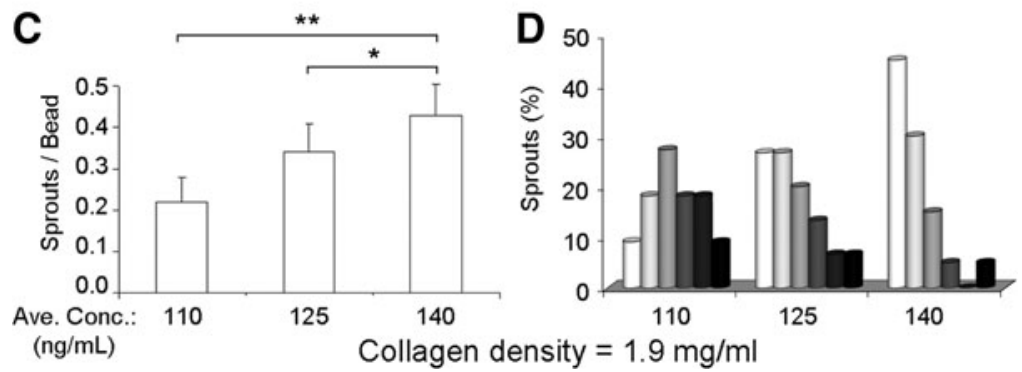

FIG. 7. Quantification of sprouts in VEGF Profile 1 and Zones I-III. (A, C) Number of sprouts formed within each Zone in 1.2 and $1.9 \mathrm{mg} / \mathrm{mL}$ collagen matrices, respectively. (B, D) Final elongation angle $\left(\theta_{2}\right)$ distributions within each zone in 1.2 and $1.9 \mathrm{mg} / \mathrm{mL}$ gels, respectively. For A-D, $n \geq 4$ microdevices per condition, each containing $\sim 20$ beads, 4 days culture; ${ }^{*} p<0.05 ;{ }^{* *} p<0.01$.

compared with the experiment duration of 4 days. ${ }^{19}$ Since fibronectin has been widely reported as enhancing VEGF activity toward ECs and has the potential to bind VEGF to the matrix, ${ }^{49,50}$ fibronectin concentration was kept constant across all matrix formulations. To assess potential VEGF binding to the biomaterial, an equilibrium gradient of Texas Red-conjugated dextran (which is not expected to bind to the matrix) was compared with the equilibrium gradient of FITC-conjugated VEGF. The two gradients were identical (Fig. 1), thus demonstrating that VEGF is not appreciably substrate bound in this biomaterial formulation. In addition, since the microfluidic device was constantly perfused with fresh medium and had a cross-sectional height and width $(240 \mu \mathrm{m} \times 1 \mathrm{~mm})$ less than the diffusion limit of tissueengineered constructs, ${ }^{51}$ it is unlikely that any observed differences in EC sprouting were due to changes in diffusivity. This is an important consideration, as recent experiments have reported that matrix-restricted diffusion can affect EC sprouting in some systems. ${ }^{17}$

In addition to diffusivity effects, changes in collagen density had a profound impact on matrix mechanics. As previously reported, increasing collagen density resulted in stiffer matrices with increased plateau storage moduli. ${ }^{19}$ Changes in matrix mechanics have been widely reported as altering cell spreading, $^{52}$ migration, ${ }^{53}$ proliferation, ${ }^{54}$ gene expression, ${ }^{55}$ and differentiation. ${ }^{56}$ Although many of these studies have been performed on carefully controlled substrates (such as 2D, amorphous polyacrylamide or PDMS gels) that allow mechanics to be altered in a manner which leaves other material properties relatively unchanged, ${ }^{56}$ as scientists look to apply these insights to more physiologically relevant biomaterials, the ability to tailor single variables will become more complex. For example, variations in collagen density can also result in substantial changes in matrix morphology, with increasing collagen concentration causing greater fiber density and greater fibril clustering into multi-stranded fibers. ${ }^{57,58}$ Both fiber dimensions and alignment have been reported as affecting cell morphology and function. ${ }^{59,60}$

In addition to effects on matrix mechanics and fiber morphology, increasing collagen density also results in an increased concentration of collagen cell-binding ligands and a possible alteration in the spatial presentation of those ligands. ECs reportedly interact with collagen cell-binding ligands via $\alpha_{1} \beta_{1}$ and $\alpha_{2} \beta_{1}$ integrin receptors. ${ }^{61}$ These interactions mediate EC sprout formation in collagen matrices by suppressing cyclic AMP and decreasing protein kinase A activation. ${ }^{61}$ Further, although the overall fibronectin concentration was held constant in all matrix formulations, and, hence, the concentration of fibronectin cell-binding ligands was also constant, the distribution and availability of those ligands may be altered due to the changes in matrix morphology. Numerous reports have detailed the ability of ligand concentration and distribution to control several cellular behaviors including cell spreading, ${ }^{62}$ migration, ${ }^{63}$ proliferation, ${ }^{64}$ gene expression, ${ }^{65}$ and differentiation. ${ }^{66}$

Given the inherent complexity of cell-biomaterial interactions in $3 \mathrm{D}$, the development of new experimental platforms that enable direct, real-time visualization of cellmaterial dynamics offers an ability to perform highthroughput analyses of different biomaterial formulations. In the studies presented here, the dynamic sprout pathfinding processes of initiation, early elongation, and navigational turning were followed over 4 days. Our experiments demonstrated that endothelial sprouts alter their pathfinding sensitivity to VEGF depending on the matrix density. At higher matrix densities, EC sprouts were more sensitive to the local VEGF concentration both during sprout initiation (Fig. 4) and sprout elongation (Figs. 5-7). In general, steeper VEGF gradients and higher VEGF concentrations were required to induce directionality and proper pathfinding in denser matrices. Steeper VEGF gradients may initiate greater sub-cellular localization of intracellular cascades that lead to subsequent asymmetry in cytoskeletal reorganization and cell polarization. ${ }^{38}$ Recent studies have also shown that the level of VEGFR2 transcription can be mediated by matrix stiffness ${ }^{14}$; therefore, the number of VEGFRs may be altered for various collagen densities, hence regulating VEGF sensitivity in a matrix-dependent manner. This hypothesis is consistent with our observation that the VEGF activation and saturation thresholds are increased in higher density 
matrices (Figs. 6 and 7), thus suggesting that VEGFR2 may be up-regulated during sprout pathfinding within stiffer matrices. Future experiments will quantitatively assess VEGFR2 presentation on tip and stalk cells in various 3D microenvironments.

Finally, we observed and quantified VEGF-induced turning of EC sprouts, a phenomenon that has not been previously described in 3D in vitro models of angiogenesis to the best of our knowledge (Fig. 3). In lower density matrices, sprouts that had originally misaligned were able to turn and properly reorient parallel to the VEGF gradient; in contrast, this turning phenomenon was only rarely observed in higher density matrices (Fig. 5). These results suggest that only a narrow range of ECM environments may be permissive to allow significant VEGF-induced sprout turning. In our previous studies, we observed statistically significant morphological differences between sprouts formed in 1.2 versus $1.9 \mathrm{mg} / \mathrm{mL}$ density matrices. ${ }^{19}$ Sprouts in lower density matrices were significantly thinner, contained fewer cells per cross-sectional area, were less likely to form a hollow lumen, and displayed faster sprouting speeds. ${ }^{19}$ This is an indication of the ability of the matrix to control the rate of new sprout formation, potentially due to its control over migration speed of the cells in 3D. It has been previously shown that denser matrices require greater proteolytic activity to induce matrix remodeling, which is a prerequisite for $3 \mathrm{D}$ cell migration. ${ }^{67,68}$ This hypothesis is consistent with our observation that cells on beads embedded in denser matrices $(1.9$ and $2.7 \mathrm{mg} / \mathrm{mL}$ ) are restricted to $2 \mathrm{D}$ migration on the bead surfaces for the first $48 \mathrm{~h}$ of culture; whereas cells in less dense matrices $(0.7$ and $1.2 \mathrm{mg} / \mathrm{mL}$ ) have already begun $3 \mathrm{D}$ migration into the matrix within $24 \mathrm{~h}$ (Fig. 2). If cellular proliferation of stalk cells is maintained at a relatively constant rate regardless of matrix density, then slower growing sprouts will result in a higher number of stalk cells per cross-sectional area. Future experiments will quantitatively assess the action of various proteases during sprout pathfinding and the migration and proliferation rates of tip and stalk cells, respectively.

Once a sprout has formed, the ability to perform a turn in direction may be related to the sprout morphology. Thicker sprouts, which are more likely to form in the higher density matrices, ${ }^{19}$ require the coordinated motion of a greater number of cells per cross-sectional area compared with thinner sprouts. Further, since thicker sprouts are more likely to have organized to form a stable, hollow lumen, ${ }^{19}$ it is possible that the mechanical requirements to induce sprout turning may be increased in higher density matrices. These hypotheses are consistent with our observations that sprout turning is more likely to occur in lower density matrices (Fig. 5).

\section{Conclusion}

In summary, we quantitatively compared 3D EC sprout navigation within various biomaterials subjected to three equilibrium VEGF concentration gradients by using an in vitro platform that allows real-time observation of sprout dynamics. This unique platform enabled visualization of tip cell filopodia extension and subsequent VEGF-induced sprout turning. Within matrices of collagen/fibronectin blends, altering the collagen density was found to result in altered sprout initiation kinetics and sprout pathfinding behavior. In general, denser matrices required steeper VEGF gradients and higher VEGF concentrations to induce proper sprout alignment and navigation. These results suggest that the optimal VEGF concentration to induce angiogenesis-like network formation in a biomaterial will be greatly dependent on the choice of scaffold; therefore, no single VEGF concentration profile will support sprouting morphogenesis for all applications. This microfluidic device will be useful in performing high-throughput screens of multiple biomaterial formulations for pro- or anti-angiogenic potential. In addition, since this platform allows separate control of soluble biochemical gradients and biomaterial properties, it may be helpful in evaluating EC-matrix interactions in a reductionist manner. Elucidating the mechanisms involved in EC sprouting morphogenesis and navigation can potentially suggest new treatments for supporting or preventing blood vessel development for tissue regeneration and cancer therapies, respectively.

\section{Acknowledgments}

The authors acknowledge funding from NIH $1 \mathrm{DP} 2$ OD006477, NIH 1R21 NS058600, NSF EFRI-CBE-0735551, NSF DMR-0846363, and National Academies Keck Futures Initiative CS10. HX acknowledges support from the Stanford Training Program in Mechanisms and Innovation in Vascular Disease, NIH 1T32HL098049-01A1.

\section{Disclosure Statement}

No competing financial interests exist.

\section{References}

1. Moon, J.J., and West, J.L. Vascularization of engineered tissues: approaches to promote angiogenesis in biomaterials. Curr Top Med Chem 8, 300, 2008.

2. Straley, K.S., and Heilshorn, S.C. Independent tuning of multiple biomaterial properties using protein engineering. Soft Matter 5, 114, 2009.

3. Kloxin, A.M., Kasko, A.M., Salinas, C.N., and Anseth, K.S. Photodegradable hydrogels for dynamic tuning of physical and chemical properties. Science 324, 59, 2009.

4. Langer, R., and Tirrell, D.A. Designing materials for biology and medicine. Nature 428, 487, 2004.

5. Lutolf, M.P., and Hubbell, J.A. Synthetic biomaterials as instructive extracellular microenvironments for morphogenesis in tissue engineering. Nat Biotechnol 23, 47, 2005.

6. Khademhosseini, A., and Langer, R. Microengineered hydrogels for tissue engineering. Biomaterials 28, 5087, 2007.

7. Hirschi, K.K., Skalak, T.C., Peirce, S.M., and Little, C.D. Vascular assembly in natural and engineered tissues. Ann NY Acad Sci 961, 223, 2002.

8. Moon, J.J., Lee, S.H., Hahn, M.S., Nsiah, B.A., and West, J.L. Regulation of endothelial angiogenesis and vasculogenesis in synthetic poly(ethylene glycol) hydrogels modified with biomolecules. FASEB J 21, A748, 2007.

9. Chen, X.F., Aledia, A.S., Ghajar, C.M., Griffith, C.K., Putnam, A.J., Hughes, C.C.W., and George, S.C. Prevascularization of a fibrin-based tissue construct accelerates the formation of functional anastomosis with host vasculature. Tissue Eng Part A 15, 1363, 2009.

10. McGuigan, A.P., and Sefton, M.V. Vascularized organoid engineered by modular assembly enables blood perfusion. Proc Natl Acad Sci USA 103, 11461, 2006. 
11. Patel, Z.S., and Mikos, A.G. Angiogenesis with biomaterialbased drug- and cell-delivery systems. J Biomater Sci Polym Ed 15, 701, 2004.

12. Zhang, G., Drinnan, C.T., Geuss, L.R., and Suggs, L.J. Vascular differentiation of bone marrow stem cells is directed by a tunable three-dimensional matrix. Acta Biomater 6, 3395, 2010.

13. Sun, G.M., and Gerecht, S. Vascular regeneration: engineering the stem cell microenvironment. Regen Med 4, 435, 2009.

14. Mammoto, A., Connor, K.M., Mammoto, T., Yung, C.W., Huh, D., Aderman, C.M., Mostoslavsky, G., Smith, L.E.H., and Ingber, D.E. A mechanosensitive transcriptional mechanism that controls angiogenesis. Nature 457, 1103, 2009.

15. Moon, J.J., Saik, J.E., Poche, R.A., Leslie-Barbick, J.E., Lee, S.H., Smith, A.A., Dickinson, M.E., and West, J.L. Biomimetic hydrogels with pro-angiogenic properties. Biomaterials 31, 3840, 2010.

16. Chen, R.R., Silva, E.A., Yuen, W.W., Brock, A.A., Fischbach, C., Lin, A.S., Guldberg, R.E., and Mooney, D.J. Integrated approach to designing growth factor delivery systems. FASEB J 21, 3896, 2007.

17. Ghajar, C.M., Chen, X., Harris, J.W., Suresh, V., Hughes, C.C.W., Jeon, N.L., Putnam, A.J., and George, S.C. The effect of matrix density on the regulation of 3-D capillary morphogenesis. Biophys J 94, 1930, 2008.

18. Sieminski, A.L., Was, A.S., Kim, G., Gong, H., and Kamm, R.D. The stiffness of three-dimensional ionic self-assembling peptide gels affects the extent of capillary-like network formation. Cell Biochem Biophys 49, 73, 2007.

19. Shamloo, A., and Heilshorn, S.C. Matrix density mediates polarization and lumen formation of endothelial sprouts in VEGF gradients. Lab Chip 10, 3061, 2010.

20. TessierLavigne, M., and Goodman, C.S. The molecular biology of axon guidance. Science 274, 1123, 1996.

21. Henley, J., and Poo, M. Guiding neuronal growth cones using Ca2 + signals. Trends Cell Biol 14, 320, 2004.

22. Davis, G.E., Bayless, K.J., and Mavila, A. Molecular basis of endothelial cell morphogenesis in three-dimensional extracellular matrices. Anat Rec 268, 252, 2002.

23. Haas, T.L. Endothelial cell regulation of matrix metalloproteinases. Can J Physiol Pharmacol 83, 1, 2005.

24. Sottile, J. Regulation of angiogenesis by extracellular matrix. Biochim Biophys Acta Rev Cancer 1654, 13, 2004.

25. Hughes, C.C.W. Endothelial-stromal interactions in angiogenesis. Curr Opin Hematol 15, 204, 2008.

26. Gerhardt, H., Golding, M., Fruttiger, M., Ruhrberg, C., Lundkvist, A., Abramsson, A., Jeltsch, M., Mitchell, C., Alitalo, K., Shima, D., and Betsholtz, C. VEGF guides angiogenic sprouting utilizing endothelial tip cell filopodia. J Cell Biol 161, 1163, 2003.

27. Ruhrberg, C., Gerhardt, H., Golding, M., Watson, R., Ioannidou, S., Fujisawa, H., Betsholtz, C., and Shima, D.T. Spatially restricted patterning cues provided by heparin-binding VEGF-A control blood vessel branching morphogenesis. Genes Dev 16, 2684, 2002.

28. Davis, G.E., and Senger, D.R. Endothelial extracellular matrix-biosynthesis, remodeling, and functions during vascular morphogenesis and neovessel stabilization. Circ Res 97, 1093, 2005.

29. Cao, L., and Mooney, D.J. Spatiotemporal control over growth factor signaling for therapeutic neovascularization. Adv Drug Deliv Rev 59, 1340, 2007.

30. Ferrara, N., Gerber, H.P., and LeCouter, J. The biology of VEGF and its receptors. Nat Med 9, 669, 2003.
31. Chappell, J.C., Taylor, S.M., Ferrara, N., and Bautch, V.L. Local guidance of emerging vessel sprouts requires soluble Flt-1. Dev Cell 17, 377, 2009.

32. Goerges, A.L., and Nugent, M.A. pH regulates vascular endothelial growth factor binding to fibronectin-a mechanism for control of extracellular matrix storage and release. J Biol Chem 279, 2307, 2004.

33. Mammoto, A., Sero, J.E., Mammoto, T., and Ingber, D.E. Methods for studying mechanical control of angiogenesis by the cytoskeleton and extracellular matrix. In: Cheresh, D.A., ed. Angiogenesis: In Vitro Systems. San Diego: Elsevier Academic Press Inc., 2008, p. 227.

34. Sieminski, A.L., Hebbel, R.P., and Gooch, K.J. The relative magnitudes of endothelial force generation and matrix stiffness modulate capillary morphogenesis in vitro. Exp Cell Res 297, 574, 2004.

35. Sieminski, A.L., Semino, C.E., Gong, H., and Kamm, R.D. Primary sequence of ionic self-assembling peptide gels affects endothelial cell adhesion and capillary morphogenesis. J Biomed Mater Res Part A 87A, 494, 2008.

36. Califano, J.P., and Reinhart-King, C.A. A Balance of substrate mechanics and matrix chemistry regulates endothelial cell network assembly. Cell Mol Bioeng 1, 122, 2008.

37. Reinhart-King, C.A., Dembo, M., and Hammer, D.A. The dynamics and mechanics of endothelial cell spreading. Biophys J 89, 676, 2005.

38. Shamloo, A., Ma, N., Poo, M.M., Sohn, L.L., and Heilshorn, S.C. Endothelial cell polarization and chemotaxis in a microfluidic device. Lab Chip 8, 1292, 2008.

39. Griffith, L.G., and Swartz, M.A. Capturing complex 3D tissue physiology in vitro. Nat Rev Mol Cell Biol 7, 211, 2006.

40. Bonvin, C., Overney, J., Shieh, A.C., Dixon, J.B., and Swartz, M.A. A multichamber fluidic device for 3D cultures under interstitial flow with live imaging: development, characterization, and applications. Biotechnol Bioeng 105, 982, 2010.

41. Nakatsu, M.N., Sainson, R.C.A., Aoto, J.N., Taylor, K.L., Aitkenhead, M., Perez-del-Pulgar, S., Carpenter, P.M., and Hughes, C.C.W. Angiogenic sprouting and capillary lumen formation modeled by human umbilical vein endothelial cells (HUVEC) in fibrin gels: the role of fibroblasts and Angiopoietin-1. Microvasc Res 66, 102, 2003.

42. Ghajar, C.M., Blevins, K.S., Hughes, C.C.W., George, S.C., and Putnam, A.J. Mesenchymal stem cells enhance angiogenesis in mechanically viable prevascularized tissues via early matrix metalloproteinase upregulation. Tissue Eng 12, 2875, 2006.

43. Vickerman, V., Blundo, J., Chung, S., and Kamm, R. Design, fabrication and implementation of a novel multi-parameter control microfluidic platform for three-dimensional cell culture and real-time imaging. Lab Chip 8, 1468, 2008.

44. Sudo, R., Chung, S., Zervantonakis, I.K., Vickerman, V., Toshimitsu, Y., Griffith, L.G., and Kamm, R.D. Transportmediated angiogenesis in 3D epithelial coculture. FASEB J 23, 2155, 2009.

45. Chung, S., Sudo, R., Mack, P.J., Wan, C.R., Vickerman, V., and Kamm, R.D. Cell migration into scaffolds under coculture conditions in a microfluidic platform. Lab Chip 9, 269, 2009.

46. Unger, R.E., Ghanaati, S., Orth, C., Sartoris, A., Barbeck, M., Halstenberg, S., Motta, A., Migliaresi, C., and Kirkpatrick, C.J. The rapid anastomosis between prevascularized networks on silk fibroin scaffolds generated in vitro with cocultures of human microvascular endothelial and osteoblast cells and the host vasculature. Biomaterials 31, 6959, 2010. 
47. Vernon, R.B., and Sage, E.H. A novel, quantitative model for study of endothelial cell migration and sprout formation within three-dimensional collagen matrices. Microvasc Res 57, 118, 1999.

48. Tammela, T., Zarkada, G., Wallgard, E., Murtomaki, A., Suchting, S., Wirzenius, M., Waltari, M., Hellstrom, M., Schomber, T., Peltonen, R., Freitas, C., Duarte, A., Isoniemi, H., Laakkonen, P., Christofori, G., Yla-Herttuala, S., Shibuya, M., Pytowski, B., Eichmann, A., Betsholtz, C., and Alitalo, K. Blocking VEGFR-3 suppresses angiogenic sprouting and vascular network formation. Nature 454, 656, 2008.

49. Liu, L.Y., Ratner, B.D., Sage, E.H., and Jiang, S.Y. Endothelial cell migration on surface-density gradients of fibronectin, VEGF, or both proteins. Langmuir 23, 11168, 2007.

50. Mahabeleshwar, G.H., Feng, W.Y., Reddy, K., Plow, E.F., and Byzova, T.V. Mechanisms of integrin-vascular endothelial growth factor receptor cross-activation in angiogenesis. Circ Res 101, 570, 2007.

51. Drury, J.L., and Mooney, D.J. Hydrogels for tissue engineering: scaffold design variables and applications. Biomaterials 24, 4337, 2003.

52. Ulrich, T.A., Pardo, E.M.D., and Kumar, S. The mechanical rigidity of the extracellular matrix regulates the structure, motility, and proliferation of glioma cells. Cancer Res 69, 4167, 2009.

53. Lo, C.M., Wang, H.B., Dembo, M., and Wang, Y.L. Cell movement is guided by the rigidity of the substrate. Biophys J 79, 144, 2000.

54. Grinnell, F., Zhu, M., Carlson, M.A., and Abrams, J.M. Release of mechanical tension triggers apoptosis of human fibroblasts in a model of regressing granulation tissue. Exp Cell Res 248, 608, 1999.

55. Paszek, M.J., and Weaver, V.M. The tension mounts: mechanics meets morphogenesis and malignancy. J Mammary Gland Biol Neoplasia 9, 325, 2004.

56. Engler, A.J., Sen, S., Sweeney, H.L., and Discher, D.E. Matrix elasticity directs stem cell lineage specification. Cell 126, 677, 2006.

57. Roeder, B.A., Kokini, K., Sturgis, J.E., Robinson, J.P., and Voytik-Harbin, S.L. Tensile mechanical properties of threedimensional type I collagen extracellular matrices with varied microstructure. J Biomech Eng 124, 214, 2002.

58. Wood, G.C., and Keech, M.K. Formation of fibrils from collagen solutions. 1. Effect of experimental conditionskinetic and electron-microscope studies. Biochem J 75, 588,1960
59. Long, J.L., and Tranquillo, R.T. Elastic fiber production in cardiovascular tissue-equivalents. Matrix Biol 22, 339, 2003.

60. Lee, P., Lin, R., Moon, J., and Lee, L.P. Microfluidic alignment of collagen fibers for in vitro cell culture. Biomed Microdevices 8, 35, 2006.

61. Whelan, M.C., and Senger, D.R. Collagen I initiates endothelial cell morphogenesis by inducing actin polymerization through suppression of cyclic AMP and protein kinase A. J Biol Chem 278, 327, 2003.

62. Ruoslahti, E., and Pierschbacher, M.D. New perspectives in cell-adhesion-Rgd and integrins. Science 238, 491, 1987.

63. Palecek, S.P., Loftus, J.C., Ginsberg, M.H., Lauffenburger, D.A., and Horwitz, A.F. Integrin-ligand binding properties govern cell migration speed through cell-substratum adhesiveness. Nature 385, 537, 1997.

64. Meredith, J.E., Jr., Fazeli, B., and Schwartz, M.A. The extracellular matrix as a cell survival factor. Mol Biol Cell 4, 953, 1993.

65. Chen, C.S., Mrksich, M., Huang, S., Whitesides, G.M., and Ingber, D.E. Geometric control of cell life and death. Science 276, 1425, 1997.

66. Kong, H.J., Boontheekul, T., and Mooney, D.J. Quantifying the relation between adhesion ligand-receptor bond formation and cell phenotype. Proc Natl Acad Sci USA 103, 18534, 2006.

67. Zaman, M.H., Trapani, L.M., Siemeski, A., MacKellar, D., Gong, H., Kamm, R.D., Wells, A., Lauffenburger, D.A., and Matsudaira, P. Migration of tumor cells in 3D matrices is governed by matrix stiffness along with cell-matrix adhesion and proteolysis. Proc Natl Acad Sci USA 103, 13897, 2006.

68. Ulrich, T.A., Jain, A., Tanner, K., MacKay, J.L., and Kumar, S. Probing cellular mechanobiology in three-dimensional culture with collagen-agarose matrices. Biomaterials 31, $1875,2010$.

Address correspondence to: Sarah Heilshorn, Ph.D.

Department of Materials Science and Engineering Stanford University 476 Lomita Mall McCullough Building Stanford, CA 94305-4045

E-mail: heilshorn@stanford.edu

Received: June 7, 2011

Accepted: September 1, 2011

Online Publication Date: October 17, 2011 